\title{
Kawasaki disease: IncRNA Slco4a1 regulates the progression of human umbilical vein endothelial cells by targeting the miR-335-5p/POU5F1 axis
}

\author{
Jingxia Hao ${ }^{1 *}$, Yingqian Zhang ${ }^{1 "}$, Xiqing Pan ${ }^{2}$, Hua Wang ${ }^{1}$, Bo Li ${ }^{1}$, Dianping You $^{3}$ \\ ${ }^{1}$ Department of Cardiology, Hebei Provincial Key Laboratory of Pediatric Cardiovascular Disease, Hebei Province Children's Hospital, Shijiazhuang, \\ China; ${ }^{2}$ Department of Joint Surgery, The Third Hospital of Shijiazhuang, Shijiazhuang, China; ${ }^{3}$ Hebei Provincial Key Laboratory of Pediatric \\ Cardiovascular Disease, Hebei Province Children's Hospital, Shijiazhuang, China \\ Contributions: (I) Conception and design: D You, J Hao; (II) Administrative support: J Hao, Y Zhang, X Pan; (III) Provision of study materials or \\ patients: None; (IV) Collection and assembly of data: None; (V) Data collection and analysis: H Wang, B Li; (VI) Manuscript writing: All authors; (VI) \\ Final approval of manuscript: All authors. \\ \#These authors contributed equally to this work and should be regarded as co-first authors. \\ Correspondence to: Dianping You. Hebei Province Children's Hospital, \#133 Jianhua Nandajie, Shijiazhuang 050031, China. \\ Email: Youdianping666@163.com.
}

Background: Kawasaki disease (KD) is an autoimmune disease with systemic vasculitis as the main pathological change, and is most common in children under 5. The role of long non-coding RNAs (lncRNAs) in human diseases has been highlighted. LncRNA Slco4a1 was reported to promote cell growth and act as an oncogenic regulator in cancer. However, the role of lncRNA Slco4a1 in KD remains unclear. This study aimed to investigate the role and mechanism of lncRNA Slco4a1 in KD.

Methods: Enzyme linked immunosorbent assay (ELISA), qRT-PCR, Western blot, and terminal deoxynucleotidyl transferase-mediated dUTP-biotin nick end labeling (TUNEL) staining were conducted to explore the function of lncRNA Slco4a1. The interaction between POU5F1 and miR-335-5p was analyzed by the RIP assay and dual luciferase assay.

Results: LncRNA Slco4a1 was significantly upregulated in the serum of KD patients compared with healthy controls. LncRNA Slco4a1 was upregulated in human umbilical vein endothelial cells (HUVECs) stimulated with KD serum. LncRNA Slco4a1 overexpression could promote the expression of inflammatory factors and apoptosis in HUVECs. The number of inflammatory cells and the infiltration area of the coronary artery in KD rats were decreased after lncRNA Slco4a1 silencing. Furthermore, lncRNA Slco4a1 is a sponge of miR-335-5p and negatively regulated the expression of miR-335-5p. POU5F1 was the downstream target of miR-335-5p, and miR-335-5p overexpression could upregulate the expression of POU5F1. Additionally, miR-335-5p overexpression could inhibit the expression of inflammatory factors and apoptosis in HUVECs. We further investigated the effect of lncRNA Slco4a1 on the mitogen-activated protein kinase (MAPK) signaling pathway, and the results showed that lncRNA Slco4al could promote the activation of the MAPK signaling pathway.

Conclusions: Together, these results indicated that lncRNA Slco4a1 could regulate the progression of HUVECs in KD by targeting the miR-335-5p/POU5F1 axis, providing new insights for KD treatment.

Keywords: Human umbilical vein endothelial cells; Kawasaki disease (KD); lncRNA Slco4a1; miR-335-5p; POU5F1

Submitted Nov 12, 2021. Accepted for publication Feb 21, 2022.

doi: $10.21037 /$ tp-22-7

View this article at: https://dx.doi.org/10.21037/tp-22-7 


\section{Introduction}

Kawasaki disease (KD), also known as Kawasaki syndrome, is an autoimmune disease with systemic vasculitis as the main pathological change, and mainly occurs in infants and young children. The main clinical features of $\mathrm{KD}$ are persistent fever, bulbar conjunctival congestion, changes of the lips and oral mucosa, dandruff of the fingers and feet, and skin rash. The pathological changes of vasculitis may involve small blood vessels, middle muscular arteries, and various organs of the body, especially the coronary arteries. This can result in coronary artery abnormalities (CAAs) including aneurysms, myocardial fibrosis, and aortic root dilation (1-3).

The molecular mechanisms underlying KD remain unclear. The hypotheses regarding the molecular mechanisms of KD are listed as follows: inflammatory factors involved in coronary artery injury and remodeling, molecular abnormalities in immune signal transduction pathways, endothelium injury, and metabolic disorders of the cell matrix, among others (4-6). In addition, some researchers have shown that long non-coding RNAs (lncRNAs) may be involved in the progression of $\mathrm{KD}$ by regulating their targets (7-9).

LncRNAs are defined as non-coding RNAs (ncRNAs) with more than 200 nucleotides that lack protein-coding ability. LncRNAs have been revealed to be involved in multiple physiological and pathological processes, including cell apoptosis, cell proliferation, and inflammation, and have been reported to play important roles in the initiation and progression of diverse diseases $(10,11)$. For example, lncRNA AK088388 was reported to promote cardiomyocyte autophagy and damage by sponging miR-30a (12). In addition, microRNAs (miRNAs) have been demonstrated to be involved in multiple diseases. For example, miR-186 was reported to facilitate endothelial cell apoptosis in $\mathrm{KD}$ by targeting SMAD6 (13). Various studies have demonstrated that the expression levels of certain mRNAs, lncRNAs, and miRNAs were upregulated in $\mathrm{KD}$ at the acute stage $(14,15)$. However, the identities and putative functions of transcripts in $\mathrm{KD}$ remain unclear. In addition, the profiles of $\operatorname{lncRNAs}$ in $\mathrm{KD}$ need further investigation.

LncRNA Slco4a1 has been reported to act as an oncogene in colorectal cancer, and could promote colorectal cancer cell growth by activating the $\beta$-catenindependent Wnt pathway (16). Furthermore, IncRNA Slco4a1 could facilitate the invasion of bladder cancer cells and upregulated OCT4 expression by sponging miR-335-
$3 p$ in bladder cancer (17). However, the role of lncRNA Slco4a1 in KD has not yet been reported. Therefore, in this study, we analyzed the role of $\operatorname{lncRNA}$ Slco $4 \mathrm{a} 1$ in KD and investigated the possible mechanisms underlying the involvement of lncRNA Slco4a1 in the regulation of KD. Differential lncRNA was screened from clinical serum samples, our study intended to explore the regulation mechanism of lncRNA Slco4a1 targeting miR-335-5p in Kawasaki disease at the cellular level.

We present the following article in accordance with the ARRIVE reporting checklist (available at https:// tp.amegroups.com/article/view/10.21037/tp-22-7/rc).

\section{Methods}

\section{Clinical specimens}

The serum samples of $32 \mathrm{KD}$ patients (16 males and 16 females; mean age: 1.72 years old), 25 healthy people (12 males and 13 females; mean age: 1.81 years old), and 17 common febrile patients ( 9 males and 8 females; mean age: 2.05 years old) were obtained from Hebei Province Children's Hospital. All $32 \mathrm{KD}$ patients met the diagnostic criteria for KD. Among the $32 \mathrm{KD}$ patients, 21 were in the acute $\mathrm{KD}$ stage and 11 were in the recovery stage. All procedures performed in this study involving human participants were in accordance with the Declaration of Helsinki (as revised in 2013). The study was approved by ethics board of Hebei Province Children's Hospital (No. 2017005) and written informed consent was obtained from the patients' guardians.

\section{Animals}

The animals used in our experiments were $8-10$ weeks old adult male Sprague Dawley rats, which were obtained from Beijing Vital River Laboratory Animal Technology Co., Ltd. Experiments were performed under a project license granted by institutional ethics board of Hebei Province Children's Hospital (No. 202101), in compliance with national guidelines for the care and use of animals.

\section{KD model establishment}

The KD model was established by a single injection of $0.5 \mathrm{~mL}$ Lactobacillus casei cell wall extract (LCWE, $1 \mathrm{mg} / \mathrm{mL}$ ) intraperitoneally, while phosphate-buffered saline (PBS) injection was used as the control. The si- 
lncRNA Slco4a1 was cloned into the lentivirus vector and administered intravenously 3 days before the establishment of the KD model (tail vein injection, $200 \mu \mathrm{L}, 1 \times 10^{8} \mathrm{TU} / \mathrm{mL}$ ).

\section{Cell culture and transfection}

Human umbilical vein endothelial cells (HUVECs) were cultured with M199 medium plus 20\% serum from KD patients or healthy controls with $20 \mathrm{ng} / \mathrm{mL}$ bovine endothelial cell growth supplement added. Lipofectamine 2000 (Invitrogen) was used for cell transfection. The pcDNA3.1, pcDNA3.1/lncRNA X, small interfering RNAs (siRNAs) of negative control (NC), and si-lncRNA X were synthesized and provided by GenePharma (Shanghai, China). After incubation for 48 hours, the cells were collected for further analysis.

\section{Quantitative real-time reverse transcription PCR ( $q R T$ - PCR)}

Total RNA was isolated using the TRIzol reagent (Thermo) and reverse transcribed using SuperScript III First-Strand Synthesis SuperMix for qRT-PCR (Thermo). PCR was performed using the StepOnePlus Real-Time PCR System (Thermo). The primers used are listed as follows: lncRNA Slco4a1, forward: 5'-GGACATGCCGGTGATGAGAG-3'; reverse, 5'-GACGCAGGGGTTCAAGTCAG-3'.

\section{Western blot}

Cells or tissues were dissected and then lysed in RIPA buffer $(150 \mathrm{mM} \mathrm{NaCl}, 50 \mathrm{mM}$ Tris-HCl, 1\% Triton, $0.5 \% \mathrm{NP} 40,1 \mathrm{nM}$ PMSF). Following centrifugation at $12,000 \mathrm{rpm}$ for 30 minutes, supernatants were collected and protein concentration was quantified using a BCA Protein Assay Kit (Thermo). A total of $50 \mu \mathrm{g}$ of protein was loaded in each lane and separated on SDS-PAGE gels, then electrophoretically transferred to polyvinylidene difluoride (PVDF) membranes (MilliPore). The membranes were blocked with $5 \%$ milk for 2 hours, followed by overnight incubation at $4^{\circ} \mathrm{C}$ with the following primary antibodies diluted in blocking buffer: anti-caspase 3 (Sigma), anticaspase 9 (Sigma), anti-Bcl-2 (Sigma), anti-Bax (Sigma), anti-p-TAK1 (CST), anti-p-P38 (CST), anti-p-JNK (CST), anti-TAK1 (CST), anti-P38 (Sigma), anti-JNK (Sigma), and anti- $\beta$-actin (Abcam), and the secondary antibodies (CST) were used.

\section{Wound healing assay}

Wound healing assay was used to detect the migration of HUVECs. As previously reported, the HUVECs were seeded in 24-well plate, and the cell confluency reached over $90 \%$, the scratch was made by a pipette tip. Then the cells were cultured for $24 \mathrm{~h}$, and the scratches were observed with a microscope (Leica, Germany) to evaluate the cell migration ability.

\section{TUNEL staining assay}

Cell apoptosis was evaluated using the DeadEnd ${ }^{\mathrm{TM}}$ Fluorometric TUNEL System (Promega) according to the manufacturer's instructions. The stained HUVECs were observed by fluorescence microscopy (NIKON).

\section{ELISA}

The expression of IL-6, CCL-2, and TNF- $\alpha$ was evaluated using ELISA kits (R\&D), and ELISA was performed according to the manufacturer's instructions.

\section{Hematoxylin and eosin (HE) staining}

The tissue was dissected and fixed using $4 \%$ paraformaldehyde (PFA) at $4{ }^{\circ} \mathrm{C}$ for 12 hours. After dehydration with $70 \%, 80 \%, 90 \%, 95 \%$, and $100 \%$ ethanol, the tissue was immersed in xylene for transparency and then embedded in paraffin. The tissue was cryosectioned into $5 \mu \mathrm{m}$-thick sections and baked for 2 hours at $65^{\circ} \mathrm{C}$. For the dewaxing procedure, the slices were immersed in xylene I, xylene II, 100\% alcohol I, 100\% alcohol II, 95\% alcohol, $90 \%$ alcohol, $80 \%$ alcohol, and $70 \%$ alcohol, and the soaking times were 20 minutes, 20 minutes, 5 minutes, 5 minutes, 4 minutes, 4 minutes, 3 minutes, and 2 minutes, respectively. Subsequently, slides were stained with hematoxylin for 5 minutes, exposed to alcohol hydrochloride differentiation solution for 2 seconds, and stained with eosin for 3 minutes. The process of dehydration was as follows: the slices were immersed in $70 \%$ alcohol, $80 \%$ alcohol, $90 \%$ alcohol, $95 \%$ alcohol, $100 \%$ alcohol I, 100\% alcohol II, xylene I, and xylene II, and the soaking times were 1 minute, 1 minute, 1 minute, 1 minute, 1 minute, 1 minute, 6 minutes, and 6 minutes, 
respectively. The images were acquired by microscopy (Nikon, Japan).

\section{Bioinformatics analysis}

The lncRNABase and miRDB databases were used to predict the target miRNAs or target genes. The differentially expressed genes were screened out using GEPIA, with $\log 2|\mathrm{FC}|>2$ and adjusted $\mathrm{P}<0.01$. Venny 2.1.0 was then used to overlap the common genes from miRDB and GEPIA. These common genes were subsequently analyzed using STRING (https://string-db.org) for proteinprotein interaction analysis.

\section{Dual luciferase assay}

The segments of wild-type POU5F1 3'UTR containing the binding site of miR-335-5p and the segments of mutant POU5F1 3'UTR without the binding site of miR-335-5p were inserted into pmirGLO vectors. The recombinant vectors were co-transfected with miRNA mimic or NC into HUVECs. After transfection for 48 hours, luciferase activity was assessed with a dual-luciferase reporter assay system (Promega).

\section{Statistical analysis}

Data were presented as mean \pm SEM. All statistical analyses were conducted using SPSS 18.0 software. Student's twotailed $t$-test was used for 2 group comparisons, and one-way ANOVA was used for multigroup comparisons. $\mathrm{P}<0.05$ was considered statistically significant.

\section{Results}

\section{LncRNA Slco4a1 was upregulated in KD serum and in HUVECs stimulated with KD serum}

Initially, we investigated the correlation between lncRNA Slco4a1 and KD. The serum samples of $21 \mathrm{KD}$ patients at the acute $\mathrm{KD}$ stage, $11 \mathrm{KD}$ patients at the recovery stage, 25 healthy people, and 17 common febrile patients were collected, and lncRNA Slco4a1 expression was analyzed using qRT-PCR. The qRT-PCR results demonstrated that compared with healthy people, lncRNA Slco4a1 expression was significantly increased in $\mathrm{KD}$ patients at the acute $\mathrm{KD}$ stage and the recovery stage (Figure $1 A$ ). The qRT-PCR results also indicated that lncRNA Slco4a1 expression and
KD were highly related.

Moreover, we investigated the effect of serum cultures from KD patients or healthy controls on the expression of lncRNA Slco4a1 in HUVECs. The qRT-PCR results demonstrated that compared with the control group, the expression of lncRNA Slco4a1 was significantly increased in the KD group (Figure 1B).

\section{LncRNA Slco4a1 induced inflammasome activation and apoptosis in HUVEC and inbibited migration}

First, the effect of lncRNA Slco4a1 on the expression of inflammatory factors was analyzed. The ELISA results showed that compared with the pcDNA3.1 group, the expression levels of inflammatory factors IL-6, CCL-2, and TNF- $\alpha$ were significantly increased in the pcDNA3.1/ lncRNA Slco4a1 group. Additionally, the expression levels of IL-6, CCL-2, and TNF- $\alpha$ were significantly decreased after lncRNA Slco4a1 knockdown (Figure 2A-2C).

Furthermore, the effect of IncRNA Slco4a1 on the apoptosis of HUVECs was analyzed. TUNEL staining results showed that compared with the pcDNA3.1 group, TUNEL-positive cells were upregulated in the pcDNA3.1/ lncRNA Slco4a1 group, and lncRNA Slco4a1 knockdown could downregulate TUNEL-positive cells (Figure 2D).

We proceeded to test the effect of IncRNA Slco4al on the expression of apoptosis-related proteins. Western blot results showed that lncRNA Slco4a1 overexpression could upregulate the expression of caspase 3, caspase 9, and Bax, and downregulate the expression of Bcl-2. LncRNA Slco4a1 knockdown demonstrated the opposite effect, that is, lncRNA Slco4a1 knockdown could decrease the expression of caspase 3, caspase 9, and Bax, and increase the expression of Bcl-2 (Figure 2E). Wound healing results (Figure $2 F$ ) revealed that compared with pcDNA3.1-NC group, the migration was reduced significantly in pcDNA3.1lncRNA Slco4a 1 group. Overall, these results demonstrated that lncRNA Slco4a1 overexpression could induce inflammasome activation, promote HUVEC apoptosis and inhibit the migration.

\section{LncRNA Slco4a1 was associated with coronary artery lesion formation in $\mathrm{KD}$ rats}

The KD model was established by a single injection of LCWE intraperitoneally, and the control group received PBS injection. The si-lncRNA Slco4a1 was administered intravenously 3 days before KD model establishment. The 


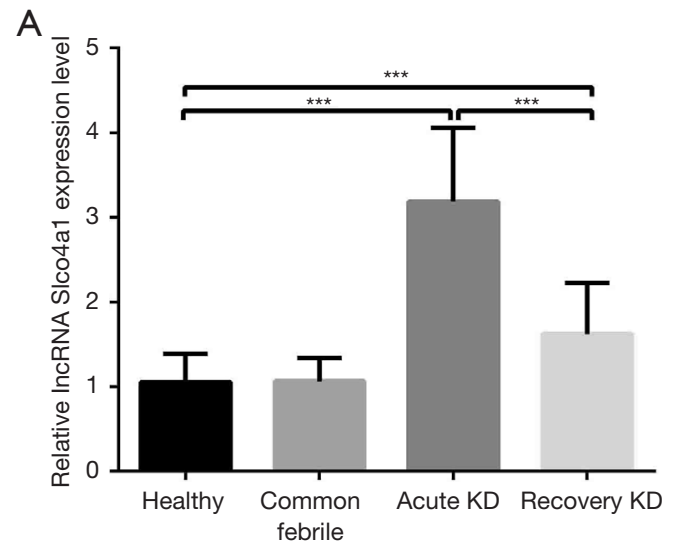

\section{B}



Figure 1 LncRNA Slco4a1 was upregulated in KD serum and in HUVECs stimulated with KD serum. (A) The expression of lncRNA Slco4a1 in the serum samples of KD patients at the acute KD stage or recovery stage, healthy people, and common febrile patients was analyzed using qRT-PCR. (B) The effect of KD patients' serum on the expression of lncRNA Slco4a1 in HUVECs was analyzed using qRTPCR. * $, \mathrm{P}<0.05,{ }^{* * *}, \mathrm{P}<0.001$. KD, Kawasaki disease; HUVECs, human umbilical vein endothelial cells.

qRT-PCR results demonstrated that the expression of lncRNA Slco4a1 was significantly decreased after si-lncRNA Slco4a 1 overexpression, validating the efficiency of silncRNA Slco4a1 lentivirus vector overexpression (Figure 3A).

Subsequently, HE staining of the coronary artery was conducted, and the representative images are shown in Figure 3B. HE staining results showed that no obvious inflammatory cell infiltration was observed in the control group, while obvious inflammatory cell infiltration was identified in the KD group. Similarly, obvious inflammatory cell infiltration was also detected in the $\mathrm{KD}+\mathrm{NC}$ group. However, lncRNA Slco4a1 knockdown could alleviate inflammatory cell infiltration (Figure 3B). The inflammatory cell infiltration area was also identified. The results showed that compared with the $\mathrm{KD}+\mathrm{NC}$ group, the infiltration area was significantly decreased in the KD + si-lncRNA Slco4a1 group (Figure 3C).

\section{LncRNA Slco4a1 is a sponge of miR-335-5p}

LncRNABase was used to predict the target miRNAs of lncRNA Slco4a1, and miR-335-5p showed the highest confidence level. Furthermore, miRDB was used to predict the target genes of miR-335-5p. Genes that were screened were subsequently analyzed using STRING (https://stringdb.org) for protein-protein interaction analysis. Among them, POU5F1 showed a very high confidence level (Figure $4 A$ ). Furthermore, the dual luciferase assay demonstrated that miR-335-5p significantly reduced the fluorescence intensity of POU5F1 wild-type but not POU5F1 mutant (Figure 4B). Subsequently, we used qRT-PCR to determine the expression of miR-335-5p in HUVECs after lncRNA Slco4a1 intervention. The qRTPCR results showed that compared with the control group, the expression of miR-335-5p was significantly reduced after lncRNA Slco4a1 overexpression, while the expression of miR-335-5p was significantly upregulated after IncRNA Slco4a1 silencing (Figure 4C).

To investigate the effect of miR-335-5p on the expression of POU5F1, we transfected miR-335-5p or miR-335-5p inhibitor into HUVECs and analyzed the expression of POU5F1, the downstream target of miR-335-5p. The qRTPCR results showed that compared with the control group, the expression of POU5F1 was significantly upregulated after miR-335-5p overexpression, while the expression of POU5F1 was significantly downregulated after miR-335-5p inhibition (Figure 4D).

\section{Effect of miR-335-5p on inflammatory factors and apoptosis in HUVECs}

Subsequently, the effect of miR-335-5p on the expression of inflammatory factors was also analyzed. ELISA results showed that the expression levels of inflammatory factors IL-6, ICAM, and IL-10 were significantly decreased after miR-335-5p overexpression, while the expression levels of IL-6, ICAM, and IL-10 were significantly increased after miR-335-5p inhibition (Figure 5A-5C). 

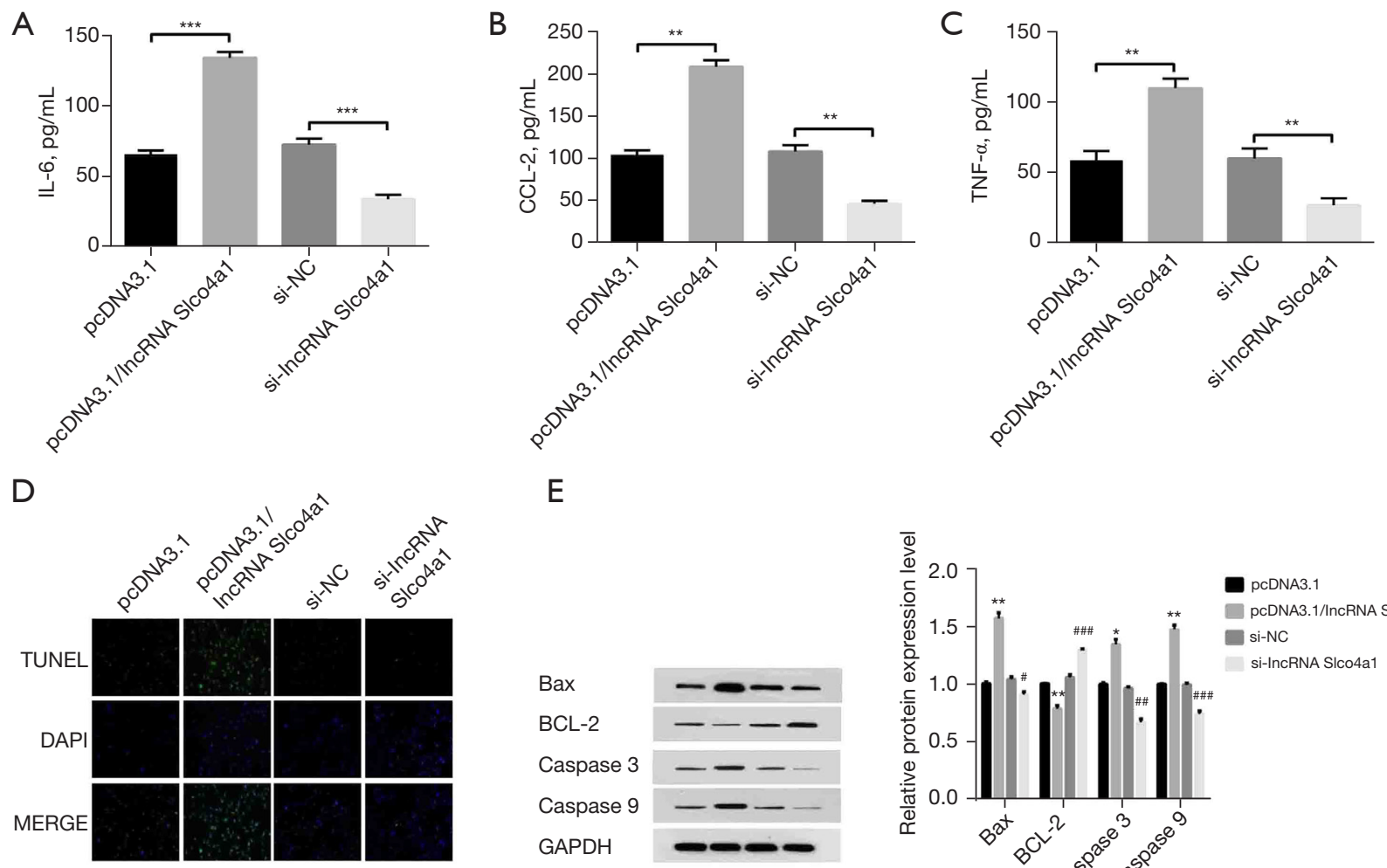

E
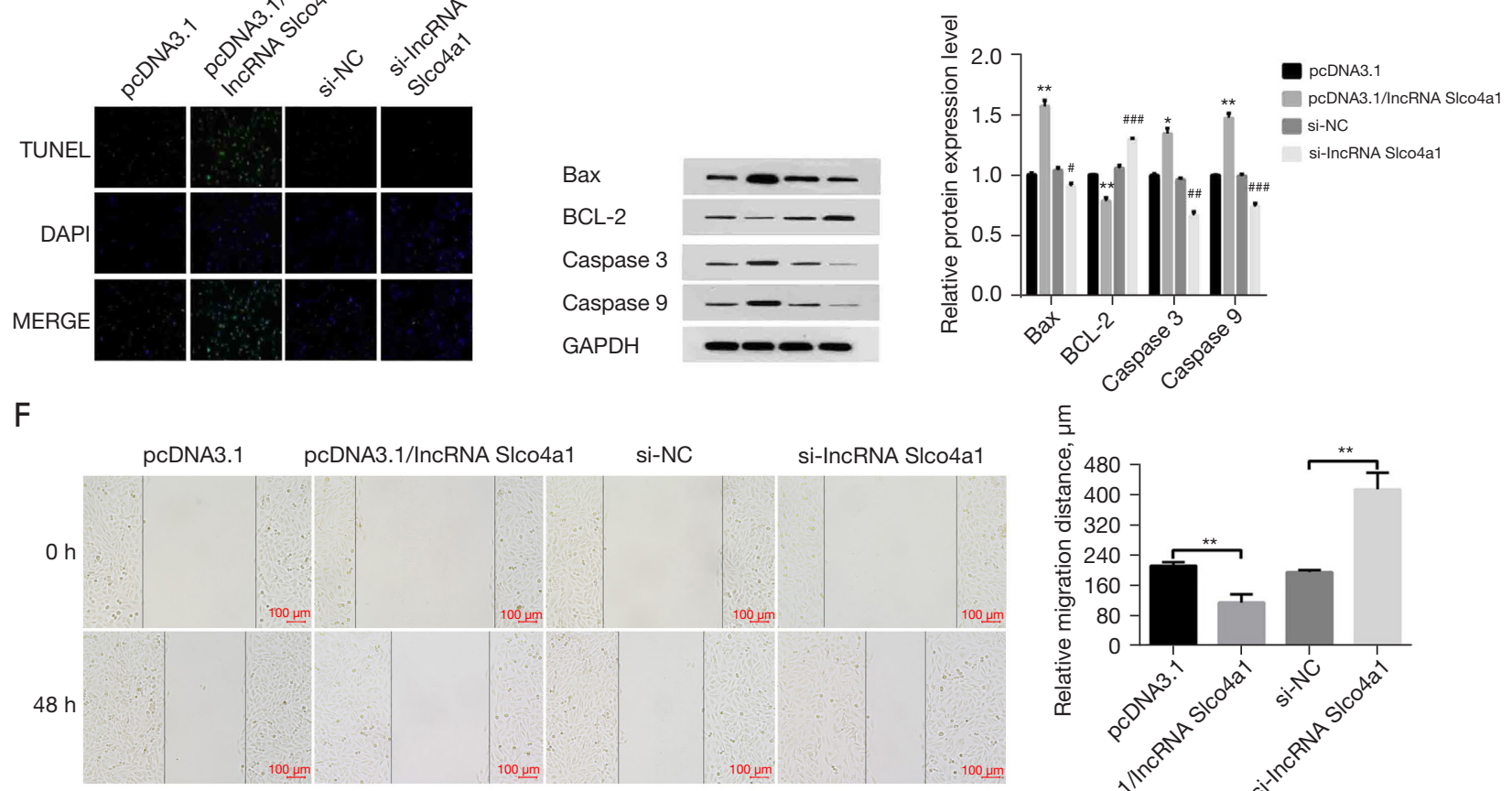

F

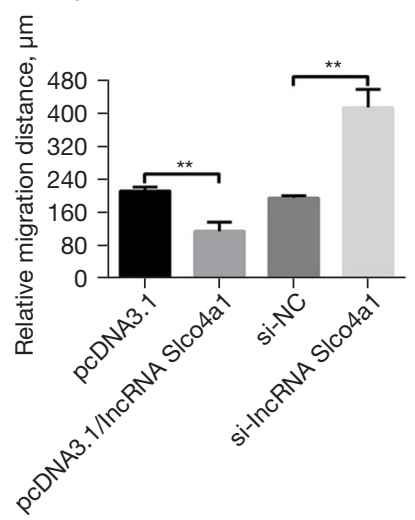

Figure 2 LncRNA Slco4a1 could promote the expression of inflammatory factors and apoptosis in HUVECs. (A-C) The effect of lncRNA Slco4a1 on the expression of IL-6, CCL-2, and TNF- $\alpha$ in HUVECs was detected using ELISA. (D) Apoptosis was analyzed using in situ TUNEL fluorescence staining. Scale bar: $20 \mu \mathrm{m}$. (E) Western blot results showed the expression of apoptosis-related proteins. (F) Wound healing assay was performed to detect the migration. Images were collected at $0 \mathrm{~h}$ and $48 \mathrm{~h}$ after injury, with the same place each time, and the migration was analyzed using Image $\mathrm{J}$ software. ${ }^{*}, \mathrm{P}<0.05,{ }^{* *}, \mathrm{P}<0.01,{ }^{* * *}, \mathrm{P}<0.001,{ }^{*}, \mathrm{P}<0.05,{ }^{\#}, \mathrm{P}<0.01,{ }^{* \#}, \mathrm{P}<0.001$. HUVECs, human umbilical vein endothelial cells; ELISA, enzyme linked immunosorbent assay; TUNEL, terminal deoxynucleotidyl transferase-mediated dUTP-biotin nick end labeling.

The effect of miR-335-5p on the apoptosis of HUVECs was also analyzed. TUNEL staining results showed that TUNEL-positive cells were downregulated after miR335-5p overexpression, while miR-335-5p inhibition could increase the expression of TUNEL-positive cells (Figure 5D).
We proceeded to test the effect of miR-335-5p on the expression of apoptosis-related proteins. Western blot results showed that miR-335-5p overexpression could upregulate the expression of $\mathrm{Bcl}-2$ and downregulate the expression of caspase 3, caspase 9, and Bax. Furthermore, 


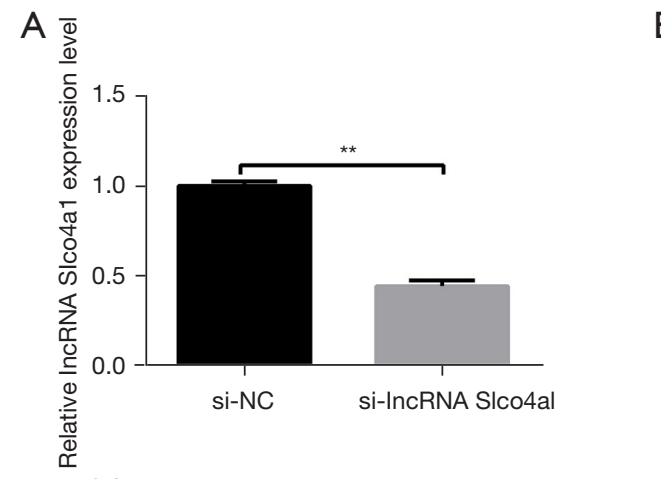

B
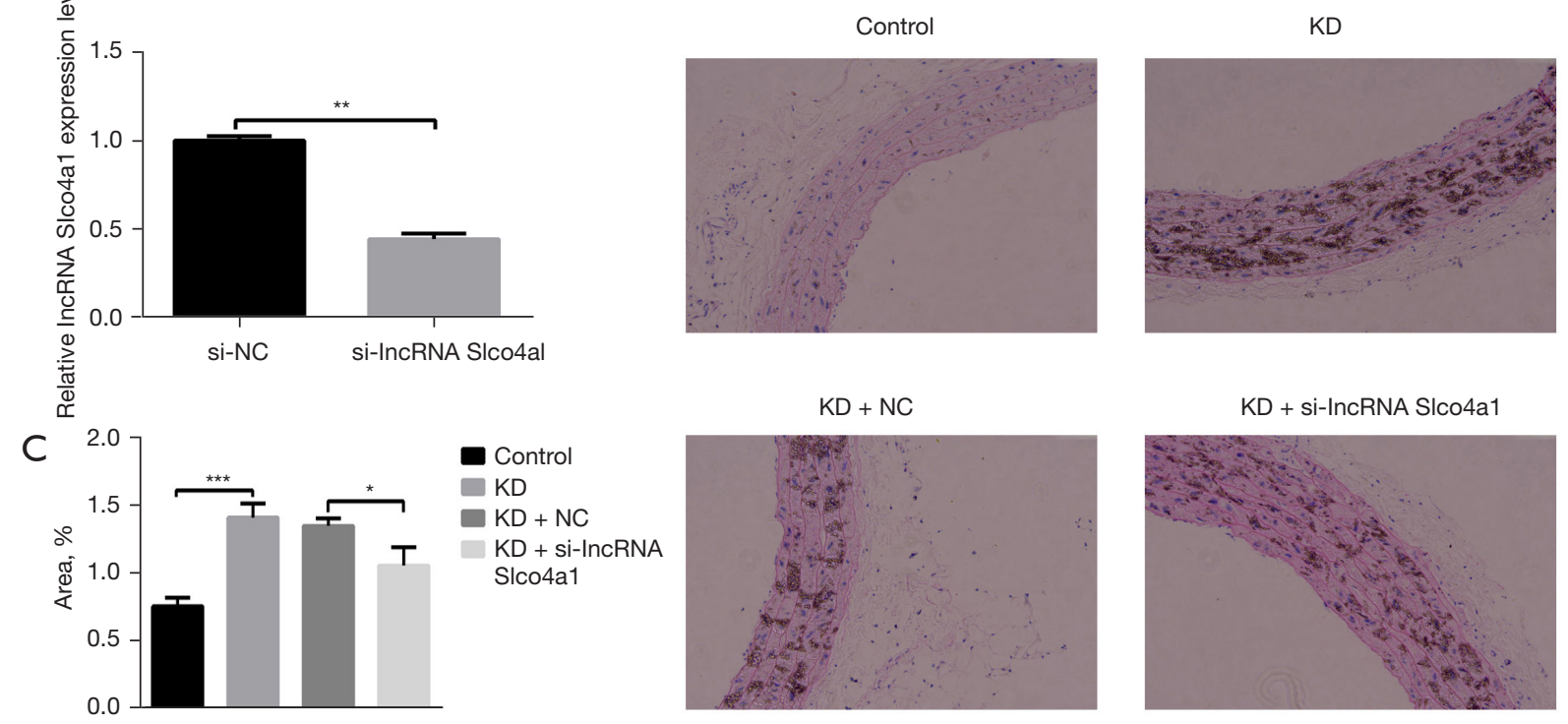

Figure 3 LncRNA Slco4a1 was associated with coronary artery lesion formation in Kawasaki disease rats. (A) The transfection efficiency of si-lncRNA Slco4a1 in vivo was verified by qRT-PCR. (B) Representative images of HE staining of the coronary artery. Scale bar: $20 \mu \mathrm{m}$. (C) The inflammatory cell infiltration area of the coronary artery was identified. * $, \mathrm{P}<0.05,{ }^{* *}, \mathrm{P}<0.01,{ }^{* * *}, \mathrm{P}<0.001$.

A

Position 185-192 of POU5F1 3' UTR

hsa-miR-335-5p

C

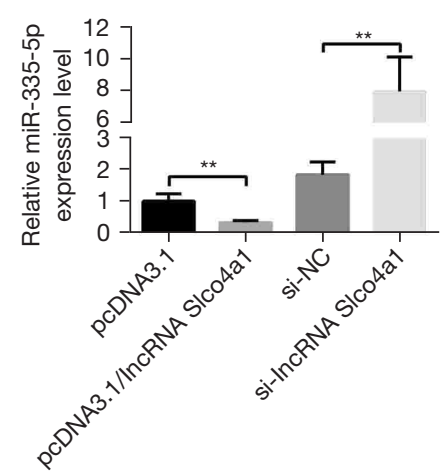

B

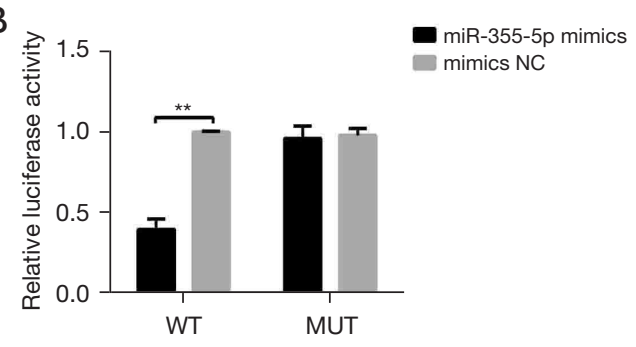

Figure 4 LncRNA Slco4a1 is a sponge of miR-335-5p. (A) The miRDB database was used to predict the target genes of miR-335-5p, and POU5F1 was the key gene connecting 3 other genes by STRING analysis. (B) The potential binding site between miR-335-5 and POU5F1 was identified using the dual luciferase assay. (C) The expression of miR-335-5 in HUVECs after lncRNA Slco4a1 intervention was evaluated using qRT-PCR. (D) The expression of POU5F1 in HUVECs transfected with miR-335-5 mimics or miR-335-5 inhibitor was evaluated using qRT-PCR. ${ }^{* *}, \mathrm{P}<0.01,{ }^{* * *}, \mathrm{P}<0.001$. HUVECs, human umbilical vein endothelial cells. 


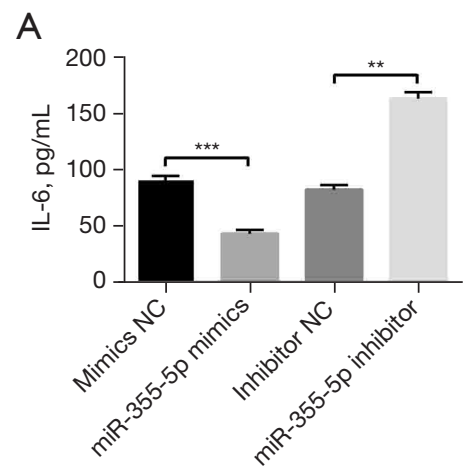

B

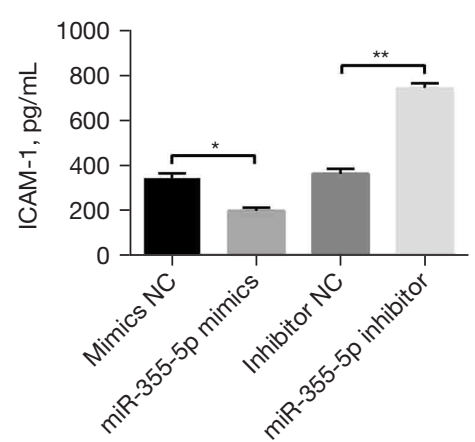

E

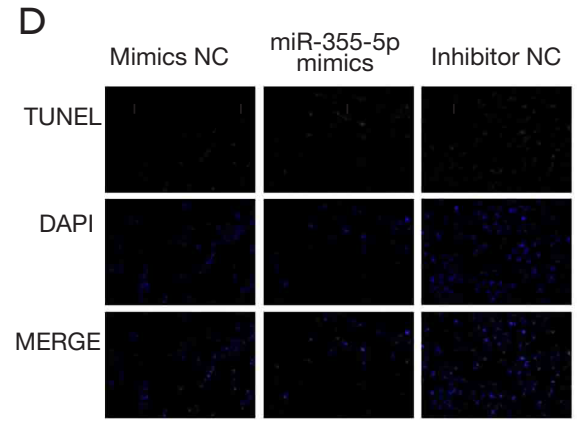

miR-355-5p
inhibitor

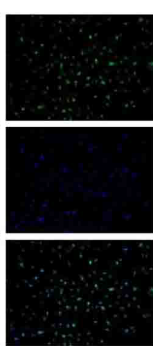

C

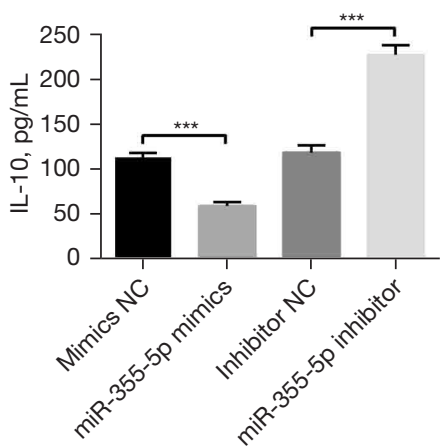

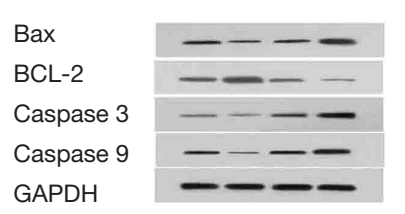

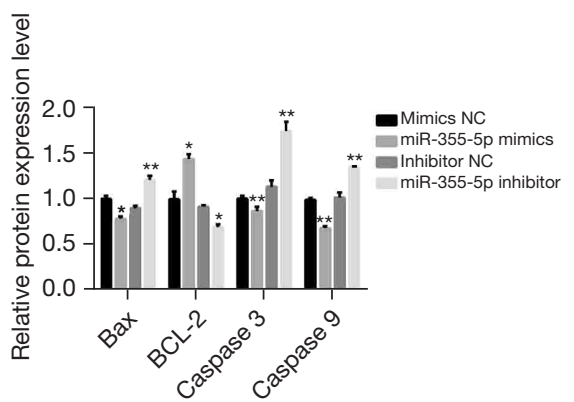

Figure 5 Effect of miR-335-5p on inflammatory factors and apoptosis in HUVECs. (A-C) The effect of lncRNA Slco4a1 on the expression of IL-6, ICAM, and IL-10 in HUVECs was detected using ELISA. (D) Apoptosis was analyzed using in situ TUNEL fluorescence staining. Scale bar: $20 \mu \mathrm{m}$. (E) The expression of apoptosis-related proteins in HUVECs transfected with miR-335-5p mimics or miR-335-5p inhibitor was evaluated using Western blot. *, $\mathrm{P}<0.05$, **, $\mathrm{P}<0.01$, ***, $\mathrm{P}<0.001$. HUVECs, human umbilical vein endothelial cells; ELISA, enzyme linked immunosorbent assay; TUNEL, terminal deoxynucleotidyl transferase-mediated dUTP-biotin nick end labeling.

miR-335-5p inhibition decreased the expression of Bcl-2 and upregulated the expression of caspase 3 , caspase 9 , and Bax (Figure 5E). These results demonstrated that miR-335$5 \mathrm{p}$ overexpression could inhibit inflammasome activation and apoptosis in HUVECs.

\section{LncRNA Slco4a1 was involved in the mitogen-activated protein kinase (MAPK) signaling pathway}

We investigated the effect of lncRNA Slco4al on the MAPK signaling pathway. The MAPK signaling pathway regulates a wide variety of cellular processes, including cell proliferation, inflammation, differentiation, and apoptosis. TAK1, P38, and JNK are important proteins involved in the MAPK signaling pathway. Western blot results showed that IncRNA Slco4a1 overexpression could upregulate the expression of p-TAK1, p-P38, and p-JNK, while lncRNA Slco4a1 silencing showed the opposite effect (Figure 6). Overall, the results indicated that lncRNA Slco4al could promote the activation of the MAPK signaling pathway.

\section{Discussion}

$\mathrm{KD}$ is an autoimmune disease with systemic vasculitis as the main pathological change. The pathological changes of vasculitis may involve various organs of the body, especially the coronary arteries, resulting in CAAs. However, the molecular mechanisms underlying $\mathrm{KD}$ remain unclear. The hypotheses regarding the molecular mechanisms underlying KD include inflammatory factor regulation in coronary artery injury and remodeling, molecular abnormalities in immune signal transduction pathways, endothelium injury, and metabolic disorders of the cell matrix, among others (4-6). In addition, lncRNAs are also involved in the progression of KD by regulating their targets. A recent study investigated the lncRNA transcript profiles of $\mathrm{KD}$ using microarray analysis and reported that XLOC_006277 was the top differentially expressed lncRNA transcript 

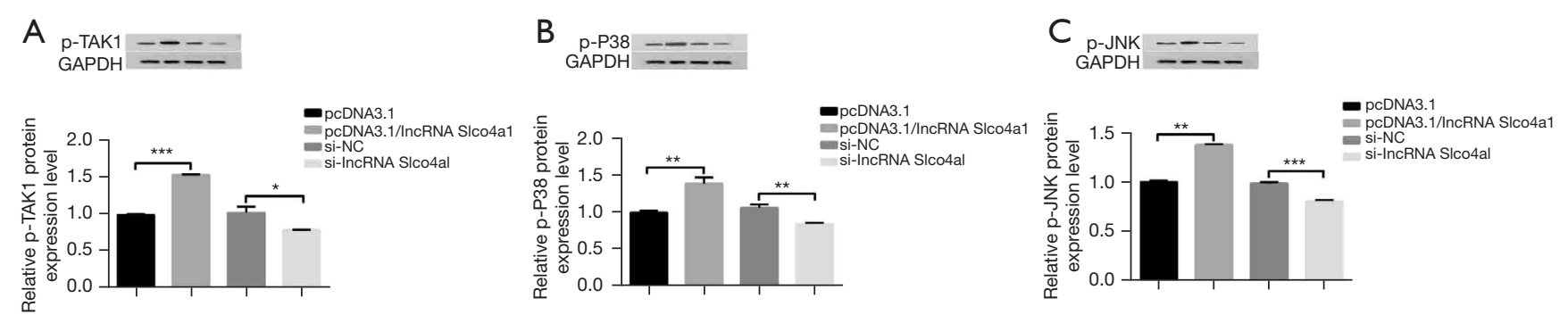

Figure 6 LncRNA Slco4a1 was involved in the MAPK signaling pathway. (A-C) Representative images of p-TAK1, p-P38, and p-JNK protein expression detected by Western blot. *, $\mathrm{P}<0.05$, **, $\mathrm{P}<0.01$, **, $\mathrm{P}<0.001$. MAPK, mitogen-activated protein kinase; TAK1, transforming growth factor- $\beta$-activated kinase 1 ; JNK, c-Jun N-terminal kinase.

among the differentially expressed lncRNA transcripts. The role of XLOC_006277 was also analyzed. XLOC_006277 knockdown could suppress MMP-8 and MMP-9 expression, which are both involved in heart lesions (18). Another study reported that miR-186 could facilitate endothelial cell apoptosis in KD by targeting SMAD6 (13). Jiang et al. (9) have reported that high expression of lncRNA PINC promotes apoptosis of vascular endothelial cells, suggesting that inhibition of IncRNA PINC may contribute to the prevention and treatment of KD. Zhao et al. (8) found that lncRNA SOCS2-AS1 up-regulated CUEDC2 by inhibiting miR-324-5p and promoted the progress of HUVECs in $\mathrm{KD}$, providing new insights for KD treatment. However, the identities and putative functions of lncRNA transcripts in $\mathrm{KD}$ remain elusive.

LncRNA Slco4a1 was reported to facilitate the growth and metastasis of colorectal cancer by $\beta$-catenin-dependent Wnt pathway activation (16). Another study reported that lncRNA Slco4a1 could promote the proliferation and metastasis of colorectal cancer via the EGFR/MAPK pathway, and could act as a potential biomarker for colorectal cancer prognosis (19). Furthermore, lncRNA Slco4a1 could facilitate cell growth in lung adenocarcinoma by modulating the miR-4701-5p/NFE2L1 axis to activate the Wnt pathway (20). The role and possible mechanism of lncRNA Slco4a1 in cancer has been well defined. However, the role of lncRNA Slco4a1 in KD has not yet been reported. Therefore, in this study, we analyzed the role of lncRNA Slco4a1 in KD, and investigated the possible mechanisms of the involvement of lncRNA Slco4a1 in the regulation of KD. We observed that lncRNA Slco4a1 was aberrantly upregulated in KD patients at the acute stage and recovery stage. We further investigated the effect of serum cultures from $\mathrm{KD}$ patients or healthy controls on the expression of lncRNA Slco4a1 in HUVECs. The results showed that treatment with KD patients' serum could upregulate the expression of lncRNA Slco4a1 in HUVECs.

We further explored the effect of lncRNA Slco4a1 on the expression of inflammatory factors. IL-6 can cause damage to vascular endothelial cells, and plays an important role in the onset and progression of hypertension $(21,22)$. In our study, we observed that the expression of IL- 6 was upregulated after lncRNA Slco4a1 overexpression in HUVECs. Inflammatory factors such as TNF- $\alpha$ have been reported to be related to $\mathrm{KD}$. The existence of vasculitis caused by a highly activated immune system in pediatric KD patients has been recognized. The apoptosis of endothelial cells induced by inflammatory cytokines may be one of the mechanisms of KD vascular injury. As an important proinflammatory factor, TNF- $\alpha$ functions by interacting with TNFR. TNF- $\alpha$ can inhibit the viability of vascular endothelial cells, and cause endothelium lesions. A previous study detected the expression of TNF- $\alpha$ in the peripheral blood mononuclear cells of pediatric patients with KD and healthy children, and reported that the expression of TNF- $\alpha$ in the KD group was significantly higher than that in the control group, suggesting that $\mathrm{TNF}-\alpha$ may play a role in the pathogenesis or progression of coronary artery lesions in KD (23). In this study, we found that the expression of TNF- $\alpha$ was upregulated after lncRNA Slco4a1 overexpression. LncRNA Slco4a1 knockdown could decrease the upregulated expression of inflammatory factors, suggesting that lncRNA Slco4a1 knockdown could inhibit coronary artery lesions in KD. Furthermore, the effect of lncRNA Slco4al on the apoptosis of HUVECs was analyzed. LncRNA Slco4a1 knockdown could inhibit the apoptosis of HUVECs.

In addition, the effect of lncRNA TUG1 knockdown on coronary artery lesions was also explored in $\mathrm{KD}$ rats. 
Obvious inflammatory cell infiltration was detected in KD rats, however, lncRNA Slco4a1 knockdown could alleviate inflammatory cell infiltration in KD rats. We further investigated the mechanism underlying the effect of lncRNA Slco4a1 on coronary artery lesions. LncRNAs are reported to interact with miRNAs and further reduce the binding of endogenous miRNAs to target genes at the post-transcriptional level. A recent study reported that lncRNA Slco4al could promote the growth and invasion of bladder cancer by sponging miR-335-5p (17). LncRNA Slco4a1 knockdown could regulate cell proliferation and apoptosis via the JNK signaling pathway (24). In this study, we observed that lncRNA Slco4a1 could positively regulate the post-transcriptional expression of POU5F1 through miR-335-5p. POU5F1 is a transcription factor belonging to the POU transcription factor family. POU5F1 can regulate the expression of target genes by binding to the OCTA Motif ATGCAAAT in the promoter or enhancer regions. The expression of POU5F1 was shown to be related to the pluripotency of stem cells and was an important factor in controlling the early stages of mammalian embryonic development (25). POU5F1 was also found to be a key gene for ensuring the orderly tissue differentiation and ontogeny of mammals during embryonic development (26).

Moreover, the effect of lncRNA Slco4a1 on the MAPK signaling pathway was also analyzed. The MAPK signaling pathway regulates a wide variety of cellular processes, including cell proliferation, inflammation, differentiation, and apoptosis $(27,28)$. The results showed that lncRNA Slco4al could promote the activation of the MAPK signaling pathway. Our findings implied that lncRNA Slco4a1 may be a potential therapeutic target and prognostic biomarker for $\mathrm{KD}$.

\section{Conclusions}

In summary, this study revealed the involvement of the lncRNA Slco4a1/miR-335-5p/ POU5F1 axis in KDinduced HUVEC injury. LncRNA TUG1 could positively regulate the post-transcriptional expression of POU5F1 through downregulating the expression of miR-335-5p, and lncRNA Slco4a1 inhibition could relieve the inflammation and apoptosis of HUVECs in KD.

\section{Acknowledgments}

We are grateful to all participants for their contributions to the present study.
Funding: None.

\section{Footnote}

Reporting Checklist: The authors have completed the ARRIVE reporting checklist. Available at https:// tp.amegroups.com/article/view/10.21037/tp-22-7/rc

Data Sharing Statement: Available at https://tp.amegroups. com/article/view/10.21037/tp-22-7/dss

Conflicts of Interest: All authors have completed the ICMJE uniform disclosure form (available at https://tp.amegroups. com/article/view/10.21037/tp-22-7/coif). The authors have no conflicts of interest to declare.

Ethical Statement: The authors are accountable for all aspects of the work in ensuring that questions related to the accuracy or integrity of any part of the work are appropriately investigated and resolved. All procedures performed in this study involving human participants were in accordance with the Declaration of Helsinki (as revised in 2013). The study was approved by ethics board of Hebei Province Children's Hospital (No. 2017005). Written informed consent was taken from all the patients' guardians. Experiments were performed under a project license granted by institutional ethics board of Hebei Province Children's Hospital (No. 202101), in compliance with national guidelines for the care and use of animals.

Open Access Statement: This is an Open Access article distributed in accordance with the Creative Commons Attribution-NonCommercial-NoDerivs 4.0 International License (CC BY-NC-ND 4.0), which permits the noncommercial replication and distribution of the article with the strict proviso that no changes or edits are made and the original work is properly cited (including links to both the formal publication through the relevant DOI and the license). See: https://creativecommons.org/licenses/by-nc-nd/4.0/.

\section{References}

1. Rife E, Gedalia A. Kawasaki Disease: an Update. Curr Rheumatol Rep 2020;22:75.

2. Agarwal S, Agrawal DK. Kawasaki disease: etiopathogenesis and novel treatment strategies. Expert Rev Clin Immunol 2017;13:247-58.

3. Sundel RP. Kawasaki disease. Rheum Dis Clin North Am 
2015;41:63-73, viii.

4. Noval Rivas M, Arditi M. Kawasaki disease: pathophysiology and insights from mouse models. Nat Rev Rheumatol 2020;16:391-405.

5. Shulman ST, Rowley AH. Kawasaki disease: insights into pathogenesis and approaches to treatment. Nat Rev Rheumatol 2015;11:475-82.

6. Sakurai Y. Autoimmune Aspects of Kawasaki Disease. J Investig Allergol Clin Immunol 2019;29:251-61.

7. Wu J, Zhou Q, Niu Y, et al. Aberrant expression of serum circANRIL and hsa_circ_0123996 in children with Kawasaki disease. J Clin Lab Anal 2019;33:e22874.

8. Zhao J, Chen D. Kawasaki disease: SOCS2-AS1/miR324-5p/CUEDC2 axis regulates the progression of human umbilical vein endothelial cells. Pediatr Res 2020. [Epub ahead of print]. doi: 10.1038/s41390-020-1029-9.

9. Jiang C, Fang X, Jiang Y, et al. TNF- $\alpha$ induces vascular endothelial cells apoptosis through overexpressing pregnancy induced noncoding RNA in Kawasaki disease model. Int J Biochem Cell Biol 2016;72:118-24.

10. Gupta SC, Awasthee N, Rai V, et al. Long non-coding RNAs and nuclear factor- $\kappa \mathrm{B}$ crosstalk in cancer and other human diseases. Biochim Biophys Acta Rev Cancer 2020;1873:188316.

11. Yu B, Wang S. Angio-LncRs: LncRNAs that regulate angiogenesis and vascular disease. Theranostics 2018;8:3654-75.

12. Wang JJ, Bie ZD, Sun CF. Long noncoding RNA AK088388 regulates autophagy through miR-30a to affect cardiomyocyte injury. J Cell Biochem 2019;120:10155-63.

13. Wu R, Shen D, Sohun H, et al. miR 186, a serum microRNA, induces endothelial cell apoptosis by targeting SMAD6 in Kawasaki disease. Int J Mol Med 2018;41:1899-908.

14. He M, Chen Z, Martin M, et al. miR-483 Targeting of CTGF Suppresses Endothelial-to-Mesenchymal Transition: Therapeutic Implications in Kawasaki Disease. Circ Res 2017; 120: 354-65.

15. Wang X, Ding YY, Chen Y, et al. MiR-223-3p Alleviates Vascular Endothelial Injury by Targeting IL6ST in Kawasaki Disease. Front Pediatr 2019;7:288. Erratum in: Front Pediatr 2019;7:449.

16. Yu J, Han Z, Sun Z, et al. LncRNA SLCO4A1-AS1 facilitates growth and metastasis of colorectal cancer through $\beta$-catenindependent Wnt pathway. J Exp Clin Cancer Res 2018;37:222.

17. Yang $\mathrm{Y}$, Wang F, Huang $\mathrm{H}$, et al. lncRNA SLCO4A1-AS1 promotes growth and invasion of bladder cancer through sponging miR-335-5p to upregulate OCT4. Onco Targets Ther 2019;12:1351-8.
18. Ko TM, Chang JS, Chen SP, et al. Genome-wide transcriptome analysis to further understand neutrophil activation and lncRNA transcript profiles in Kawasaki disease. Sci Rep 2019;9:328.

19. Tang R, Chen J, Tang M, et al. LncRNA SLCO4A1-AS1 predicts poor prognosis and promotes proliferation and metastasis via the EGFR/MAPK pathway in colorectal cancer. Int J Biol Sci 2019;15:2885-96.

20. Wei Y, Wei L, Li J, et al. SLCO4A1-AS1 promotes cell growth and induces resistance in lung adenocarcinoma by modulating miR-4701-5p/NFE2L1 axis to activate WNT pathway. Cancer Med 2020;9:7205-17.

21. Loperena R, Van Beusecum JP, Itani HA, et al. Hypertension and increased endothelial mechanical stretch promote monocyte differentiation and activation: roles of STAT3, interleukin 6 and hydrogen peroxide. Cardiovasc Res 2018;114:1547-63.

22. Szulcek R, Sanchez-Duffhues G, Rol N, et al. Exacerbated inflammatory signaling underlies aberrant response to BMP9 in pulmonary arterial hypertension lung endothelial cells. Angiogenesis 2020;23:699-714.

23. Cruz-Olivo F, García-Elorriaga G, González-Bonilla C, et al. Tumor necrosis factor -308 and lymphotoxin +252 polymorphisms in Mexican children with Kawasaki disease and coronary aneurysms. Arch Med Res 2011;42:602-7.

24. Mao J, Gao W, Xue L, et al. The lncRNA SLCO4A1AS1/miR-876-3p/RBBP6 axis regulates cell proliferation and apoptosis in acute lymphocytic leukemia via the JNK signaling pathway. Int J Lab Hematol 2021;43:1050-61.

25. Lee MT, Bonneau AR, Takacs CM, et al. Nanog, Pou5f1 and SoxB1 activate zygotic gene expression during the maternal-to-zygotic transition. Nature 2013;503:360-4.

26. Daigneault BW, Rajput S, Smith GW, et al. Embryonic POU5F1 is Required for Expanded Bovine Blastocyst Formation. Sci Rep 2018;8:7753.

27. Fang JY, Richardson BC. The MAPK signalling pathways and colorectal cancer. Lancet Oncol 2005;6:322-7.

28. Liao HH, Zhang N, Meng YY, et al. Myricetin Alleviates Pathological Cardiac Hypertrophy via TRAF6/TAK1/ MAPK and Nrf2 Signaling Pathway. Oxid Med Cell Longev 2019;2019:6304058.

Cite this article as: Hao J, Zhang Y, Pan X, Wang H, Li B, You D. Kawasaki disease: lncRNA Slco4al regulates the progression of human umbilical vein endothelial cells by targeting the miR335-5p/POU5F1 axis. Transl Pediatr 2022;11(2):183-193. doi: $10.21037 /$ tp $-22-7$ 